\title{
Uso de drones na atualização de área construída de imóveis urbanos
}

Use of drones in updating constructed area of urban buildings

\author{
B. H. T. Arantes ${ }^{1}$; L. T. Arantes ${ }^{2}$; M. V. A. Ventura ${ }^{1}$; E. M. Costa ${ }^{1} ;$ M. \\ M. Bessa ${ }^{3}$; L. M. Baliza ${ }^{1}$; V. H. Moraes ${ }^{1}$ \\ ${ }^{1}$ Instituto Federal Goiano, 75906-785, Rio Verde - GO, Brasil \\ ${ }^{2}$ Unesp, 15385-000, Ilha Solterira - SP, Brasil \\ ${ }^{3}$ Unesp, 17525-000, Marília - SP, Brasil
}

bhtondato@gmail.com

(Recebido em 01 de julho de 2018; aceito em 02 de outubro de 2018)

\begin{abstract}
Diante da possibilidade da utilização da geotecnologia para a atualização de área construída dos imóveis urbanos, foi analisada a precisão de uma imagem aérea obtida por meio de um Veículo Aéreo não Tripulado (VANT) sem a utilização de pontos de controle, na medição de um imóvel. Para isto, utilizou-se diversas resoluções espaciais, de maneira a comparar, qual seria o erro de cada um deles. Alcançaram-se bons resultados com o pixel de 2,5 e $10 \mathrm{~cm}$, mas, um resultado inferior na resolução espacial de $20 \mathrm{~cm}$, já que a última pode gerar algumas confusões.
\end{abstract}

Palavras chaves: Imagem aérea; Resolução espacial; Cadastro urbano.

Considering the possibility of the use of geotechnology to update the constructed area of urban properties, the accuracy of an aerial image obtained by means of an Unmanned Aerial Vehicle (UAV) was analyzed without the use of control points, in the measurement of a property. For this, several spatial resolutions were used, in order to compare, what would be the error of each of them. Good results were obtained with the 2,5 and $10 \mathrm{~cm}$ pixel, but with a lower result in the spatial resolution of $20 \mathrm{~cm}$, since the latter can generate some confusion.

Keywords: Aerial image; Spatial resolution; Urban registry.

\section{INTRODUÇÃO}

Aplicação de imagens para a atualização cadastral e para a detecção de alterações dos imóveis urbanos começou a surgir na década de 90 para o início do ano de 2000, com o surgimento de imagens orbitais de alta resolução espacial [2]. No entanto, como elas não possuem precisões melhores do que 30 centímetros, tal que a melhor resolução espacial do mercado é de 0,3 metros, elas ainda não são suficientes para medições de imóveis urbanos. Todavia, os sistemas de aviação remotamente pilotados ou não tripulados (RPAS), mais conhecidos coloquialmente como Drones [3], ganharam crescente popularidade no sensoriamento remoto, uma vez que fornece um sistema de aquisição rápido, de baixo custo e flexível para dados de alta resolução espacial e geração de ortofotos $[4,11]$. Os desenvolvimentos relacionados a eles iniciaram-se na década de 70 para fins militares, porém, hoje são inúmeras aplicações e possibilidade que eles proporcionam [6].

De forma tradicional, as informações cadastrais são realizadas por meio de trenas e medidores eletrônicos de distância, ou ainda por meio da utilização de receptores GNSS (Global Navigation Satellite System). Esses instrumentos apresentam um alto nível de precisão e desempenho no levantamento de pontos, linhas e objetos, no entanto são lentos. Em contraste com esses métodos de levantamento tradicionais, as aplicações fotogramétricas são usadas para criar e atualizar mapas ou orto-imagens, especialmente para áreas maiores, porém as imagens convencionais no ar são limitadas em sua utilização para o levantamento cadastral, em função à alta altitude de voo e das altas despesas [8].

Em contrapartida, os RPAS permitem obter imagens em resoluções iguais ou superiores aos voos tripulados, ainda sem a presença de nuvens e com um baixo custo. Quanto à precisão, 
eles atendem aos levantamentos planialtimétricos para fins de medição de área construída, permitindo a otimização na coleta dos dados com relação às variáveis tempo e custo, desde que se tenham uma imagem de resolução que permita tal precisão [9]. A precisão dos Veículos Aéreos não Tripulados (VANT) depende de alguns fatores múltiplos, tal como a qualidade da câmera, a calibração da câmera, o número e a localização dos pontos de controle na terra e a escolha do software de processamento $[7,12]$.

Ainda, de acordo com Turner et al. (2012) [13], as imagens aéreas de alta qualidade podem ser adquiridas usando plataformas convencionais, como satélites e aeronaves, mas sua resolução temporal é limitada pela disponibilidade restrita de plataformas de aeronaves e características de órbita de satélites. Logo, isso limita seu uso para fins de atualização de mapas, pois aumentará o custo e o tempo de produção. Recentemente, os RPAS foram introduzidos nas atividades de mapeamento e foram vinculados com uma produção de baixo custo de dados espaciais precisos e de alta qualidade em pouco tempo [10].

Além disso, no contexto do mapeamento cadastral contemporâneo, apesar dos limites cadastrais ainda serem detectados visualmente e digitalizados manualmente, existem algumas pesquisas que estão buscando alternativas para uma vetorização mais rápida. Como exemplo, temse o trabalho de Crommelinck et al. (2017) [5] que utilizou métodos da visão computacional para detectar contornos de imóveis rurais e de objetos físicos na imagem em regiões rurais, no qual apresentou bons resultados. Entretanto, esta técnica ainda é carente em centros urbanos.

Quanto à legislação de uso do equipamento, de acordo com Anac (2017) [1], os veículos remotamente pilotados, com mais de 250 gramas, só poderão sobrevoar em áreas distante de terceiros, de no mínimo 30 metros. No entanto, caso exija barreira de proteção entre o equipamento e as pessoas à distância especificada não precisa ser seguida. Além disto, os operadores destas aeronaves (com até $250 \mathrm{~g}$ ) são considerados licenciados, sem a necessidade de possuir documento emitido pela ANAC (Agência Nacional de Aviação Civil) desde que o equipamento não seja usado para voos acima de 400 pés. Entretanto, o uso de RPAS para órgãos de segurança pública, o voo é permitido pela ANAC, sem observar os critérios de distanciamento das áreas distante de terceiros. Para voos acima de 400 pés, é obrigatória a licença e a habilitação emitidas pela ANAC para os operadores de aeronaves não tripuladas.

Logo, a fim de superar todas as dificuldades trazidas pelos métodos tradicionais de atualização cadastral, tal pesquisa teve como objetivo demonstrar e analisar a capacidade da utilização de veículos aéreos não tripulados para fins de medição de área construída de um imóvel com a utilização de uma ortofoto sem pontos de controle. Com isto, foi realizada uma comparação dos valores da área construída obtidos por meio da imagem aérea e do real valor do imóvel.

\section{MATERIAL E MÉTODOS}

A cobertura aerofotogramétrica do local de estudo foi realizada a uma altura de voo de 30 metros a fim de garantir uma melhor qualidade das fotografias aéreas e de seus produtos subsequentes. Nas linhas de voos uma sobreposição de $85 \%$ foi adotada no sentido lateral e longitudinal. Apesar do voo de 30 metros gerar um pixel de $1 \mathrm{~cm}$, foram extraídos ortofotos com pixels de $2,5,10$ e $20 \mathrm{~cm}$.

O levantamento foi executado por um Phantom 4 Advanced equipado com um sistema de aquisição de coordenadas do centro perspectivo das fotografias, em conjunto com um sistema inercial de medida para disponibilizar o ângulo de inclinação das imagens capturadas. Para a geração das ortofotos do voo de 30 metros as imagens foram processadas pelo DroneDeploy, na qual todo o processo de junção e correção das posições e ângulos das fotografias são realizados. Como resultado tem-se uma única imagem bidimensional de toda a área levantada.

Para a avaliação do objetivo aqui proposto, os mosaicos bidimensionais foram exportados do DroneDeploy com pixels de 2, 5, 10 e $20 \mathrm{~cm}$, de maneira que cada um deles foram trabalhadas de forma separada no QGIS 2.18.14 para a medição de suas áreas construídas e para suas respectivas vetorizações.

No QGIS 2.18.14 foi realizada a vetorização para cada mosaico, das áreas construídas com beiral e sem beiral, uma vez que por meio da imagem aérea só é possível digitalizar o limite 
do imóvel com beiral, para depois descontar-se o tamanho do mesmo. Nesta pesquisa, a construção contava com um beiral de 1 metro. Após as vetorizações, as áreas foram calculadas para cada restituição gerada. A vetorização foi realizada com o auxílio das ferramentas de Digitalização e Digitalização Avançado do QGIS 2.18.14. O cálculo de área foi executado pela Calculadora de Atributos.

As medições das áreas construídas já com o beiral descontado de cada mosaico de diferentes resoluções espaciais foram comparadas com o verdadeiro valor de área do imóvel, uma vez que a cobrança do IPTU (Imposto Predial e Territorial Urbano) é feita sobre a área sem o beiral. Logo nas imagens levantadas não se adotou pontos de controle, tal que o objetivo era analisar a capacidade de se medir área construída por meio de imagens de Veículos Aéreos não Tripulados (VANTs) com a precisão de um GNSS comum, que varia em torno de 3 a 4 metros.

Através de um plano de voo semiautônomo elaborado no aplicativo do DroneDeploy para a obtenção de um pixel de $2,5,10$ e $20 \mathrm{~cm}$ seria necessário realizar um voo a 70, 170, 330 e acima de 500 metros respectivamente.

\section{RESULTADOS}

$\mathrm{Na}$ Figura 1, têm-se as restituições realizadas nas resoluções espaciais de 2 e 5 centímetros, e seus respectivos valores mensurados por meio da vetorização sem beiral e com beiral da imagem aérea.

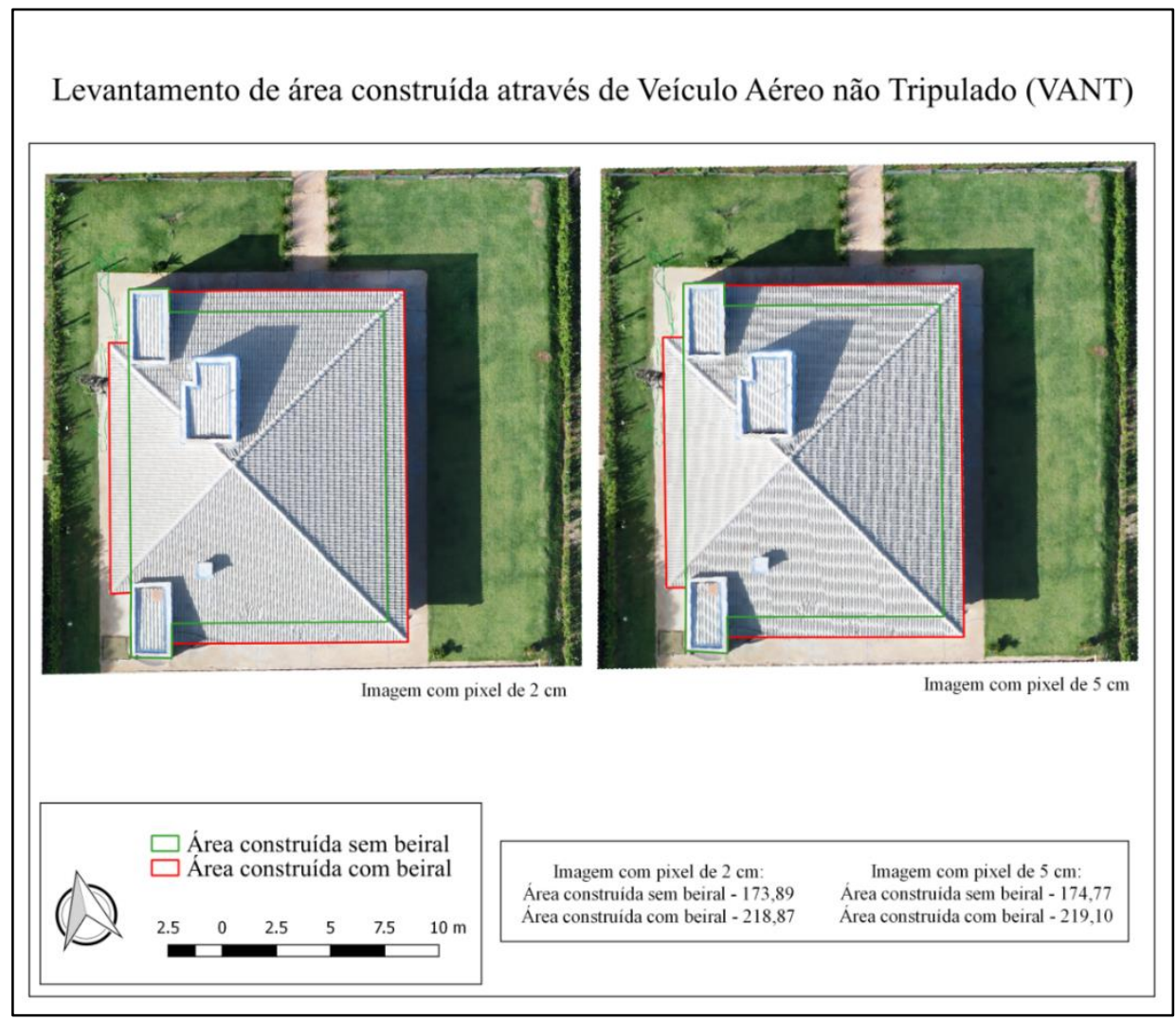

Figura 1: Mosaicos das áreas construídas com pixel de 2 e $5 \mathrm{~cm}$

Na Figura 2, têm-se as restituições realizadas nas resoluções espaciais de 10 e 20 centímetros, e o valor mensurado por meio da vetorização sem beiral e com beiral da imagem aérea para o GSD (Ground Sample Distance) de $10 \mathrm{~cm}$. 


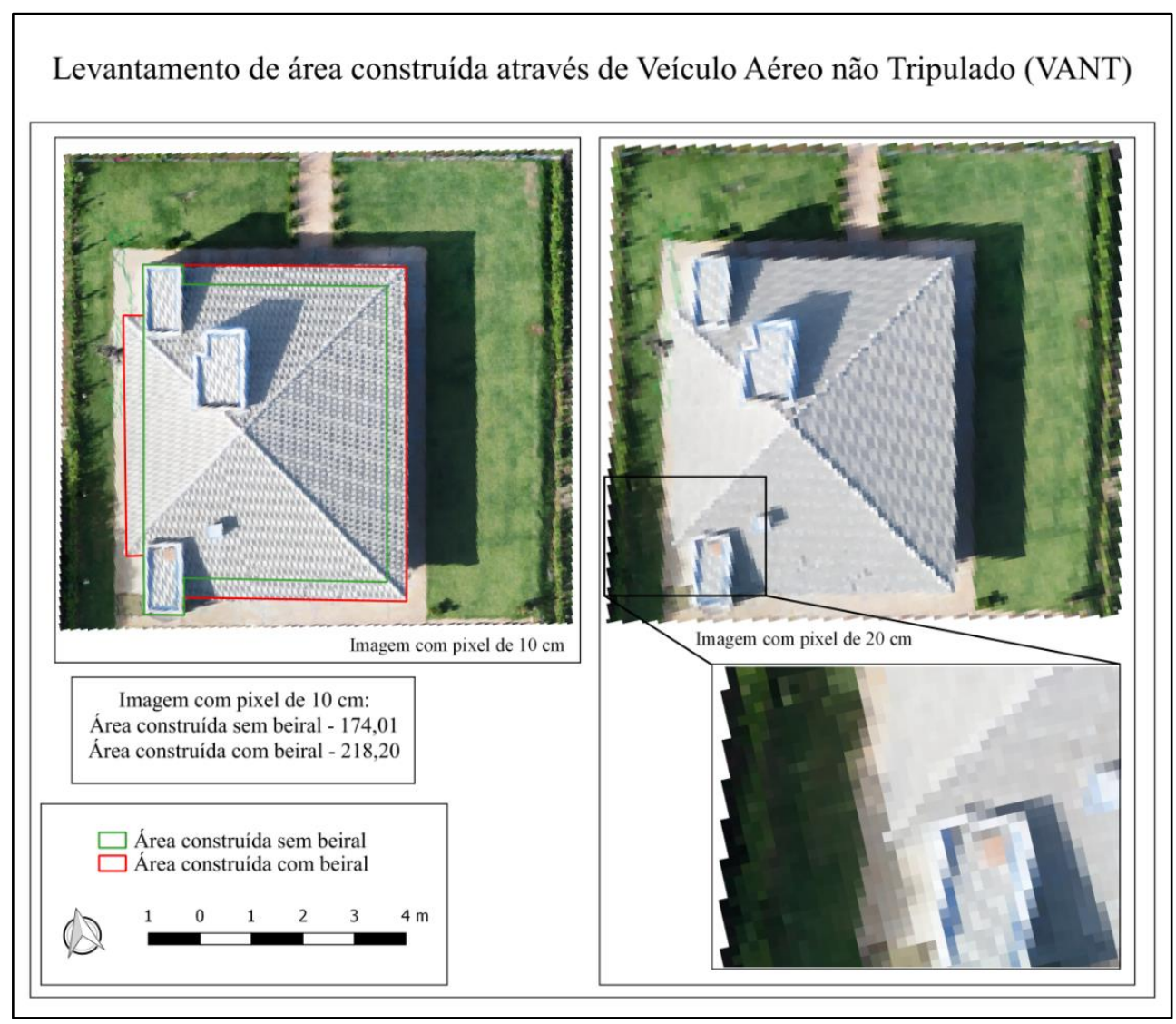

Figura 2: Mosaicos das áreas construídas com pixel de 10 e $20 \mathrm{~cm}$

Juntamente, com os valores de área construída com beiral e sem beiral, temos o valor real do imóvel que é apresentado na Tabela 1.

Tabela 1: Valores de área construída em metros quadrados

\begin{tabular}{|c|c|c|c|}
\hline Resolução espacial & $\begin{array}{l}\text { Área construída sem } \\
\text { beiral (ACSB) }\end{array}$ & $\begin{array}{l}\text { Área construída com } \\
\text { beiral }(\mathrm{ACCB})\end{array}$ & $\begin{array}{l}\text { Área construída } \\
\text { real }(\mathrm{ACR})\end{array}$ \\
\hline $2 \mathrm{~cm}$ & $173,89 \mathrm{~m}^{2}$ & $218,87 \mathrm{~m}^{2}$ & $182,93 \mathrm{~m}^{2}$ \\
\hline $5 \mathrm{~cm}$ & $174,77 \mathrm{~m}^{2}$ & $219,10 \mathrm{~m}^{2}$ & $182,93 \mathrm{~m}^{2}$ \\
\hline $10 \mathrm{~cm}$ & $174,01 \mathrm{~m}^{2}$ & $218,20 \mathrm{~m}^{2}$ & $182,93 \mathrm{~m}^{2}$ \\
\hline
\end{tabular}

Por fim, temos na Tabela 2 as diferenças encontradas entre as áreas construídas sem beiral e o real valor do imóvel para as resoluções espaciais de 2,5 e $10 \mathrm{~cm}$.

Tabela 2: Discrepância de entre o real valor da área construída e aquela obtida por meio de VANT

\begin{tabular}{ccc}
\hline Resolução espacial & ACSB - ACR & ACSB - ACR (\%) \\
\hline $2 \mathrm{~cm}$ & $9,04 \mathrm{~m}^{2}$ & 4,94 \\
$5 \mathrm{~cm}$ & $8,16 \mathrm{~m}^{2}$ & 4,46 \\
$10 \mathrm{~cm}$ & $8,92 \mathrm{~m}^{2}$ & 4,88 \\
\hline
\end{tabular}

\section{DISCUSSÃO}

Com a constante expansão das áreas urbanas e com a chegada dos VANTs se tornou possível medir áreas construídas e adequar as irregularidades nas dimensões dos imóveis urbanos em um curto intervalo de tempo e com um baixo custo. Com isto, algumas medidas foram extraídas através de mosaicos de diferentes resoluções espaciais, para inferir a precisão destas ortofotos sem pontos de controle (Figuras 1 e 2). 
Nos mosaicos com resoluções de 2, 5 e $10 \mathrm{~cm}$, o contorno do imóvel e o final do beiral podem ser identificados sem dificuldades, o que permite uma vetorização precisa de toda área construída. No entanto com uma resolução espacial de $20 \mathrm{~cm}$, a vetorização se torna duvidosa, uma vez que a calçada se confunde com o telhado em certa parte do imóvel, de acordo com a imagem expandida na Figura 2.

Os valores de medidas obtidos por meio das imagens aéreas mostram que as áreas construídas sem beiral e com beiral não variam de forma significativa entre a resolução de $2,5 \mathrm{e}$ $10 \mathrm{~cm}$, o que permite se trabalhar com uma resolução menor sem perda de precisão das medidas, otimizando o trabalho (Tabela 1). Com uma resolução de $10 \mathrm{~cm}$, uma área de 20 hectares com uma faixa de sobreposição de $80 \%$ na lateral e longitudinal poderia ser levantada em aproximadamente 10 minutos. Nas mesmas especificações, porém com uma resolução de $2 \mathrm{~cm}$, se gastaria por volta de 42 minutos.

A área construída encontrada por meio do veículo aéreo não tripulado foi menor do que o real valor do imóvel, para qualquer resolução. Além disso, devido a maior discrepância de medidas entre as diferentes resoluções espaciais ficar em 0.88 metros quadrados, e a ortofoto de $5 \mathrm{~cm}$ ter apresentado um resultado mais próximo do verdadeiro, ou seja, o melhor resultado, e a de $2 \mathrm{~cm}$ o pior, pode-se inferir que tal diferença está relacionada à acuidade visual de se vetorizar sobre o limite da construção e não a resolução espacial (Tabela 2).

A maior diferença de área encontrada entre o VANT e o real valor de área do imóvel foi de 4,94\% para menos (Tabela 2). Apesar de o valor ser considerável quando se engloba um grande número de casas, ele é ainda pequeno quando comparado ao valor que as prefeituras estão deixando de receber pela presença de áreas irregulares. Em vista da dificuldade de se conferir o valor da metragem dos imóveis de forma tradicional, o VANT permite que a atualização seja realizada de forma rápida e com intervalos de novas atualizações de acordo com a necessidade de cada município.

Contudo, para que esta técnica seja executada de forma eficiente e coerente, além de seguir à metodologia aqui estudada, o padrão de medida dos beirais deve ser conhecido para que seja descontado. Além disso, é indispensável uma visita em cada fachada das casas para que sejam identificadas as construções acima de um piso, já que na imagem aérea tal identificação não é possível.

\section{CONCLUSÃO}

Pode-se inferir que para a medição de área construída as resoluções espaciais de 2,5 e 10 $\mathrm{cm}$ apresentam bons contrastes para vetorizar ou digitalizar os limites dos imóveis. No entanto com uma resolução de $20 \mathrm{~cm}$ pode ocorrer uma confusão em telhados que apresentem a mesma tonalidade que outros objetos ou construções. Não obstante, as imagens orbitais ainda não possuem a capacidade de serem utilizadas para medições de áreas construídas, tal que a melhor resolução do mercado é de $30 \mathrm{~cm}$. No entanto testes com todas as resoluções aqui citadas podem ser feitas para medição de construções por meio da técnica de estereoscopia, para se verificar a possibilidade da utilização de resoluções iguais ou acima de $20 \mathrm{~cm}$.

A técnica aqui aplicada, apesar de não estar georreferenciada a uma precisão centimétrica, ela pode ser aplicada para medições de áreas construídas sem mesmo a utilização de pontos de controle, uma vez que a área dada pelo VANT é menor que a área real. Devido à discrepância da área real e a dada pelo VANT ser menor que 5\%, isso ainda traz um benefício à prefeitura, já que tem a possibilidade de realizar a regularização de todos os imóveis quanto aos seus tamanhos em um curto intervalo de tempo, sem a necessidade de realizar uma visita a todas as propriedades (técnica tradicional de aferição).

Com relação à legislação, a realização dos voos para a obtenção dos GSD de 2, 5, 10 e 20 centímetros, ainda não é permitido, devido à existência de um grande número de pessoas expostas ao risco de acordo com a normativa da ANAC, uma vez que a distância mínima exigida a elas na horizontal é de 30 metros. Mas, com relação à altura, o voo para a obtenção das resoluções espaciais de 5, 10 e $20 \mathrm{~cm}$, poderia ocorrer desde que o operador tenha a licença e a habilitação emitidas pela a ANAC (ANAC, 2017). Quanto ao voo para o pixel de $20 \mathrm{~cm}$, o mesmo não é 
viável para o modelo de equipamento aqui testado, tal que ele não apresenta uma autonomia de voo o suficiente para alturas maiores do que 500 metros.

\section{AGRADECIMENTOS}

A CAPES e a CNPq pelo apoio financeiro. Ao Instituto Federal Goiano e a Unesp pela honra de estar cursando o mestrado.

\section{REFERÊNCIAS BIBLIOGRÁFICAS}

1. Anac - Agência Nacional de Aviação Civil. Regras sobre Drones: Regras da ANAC para uso de drones entram em vigor. Disponível em: < http://www.anac.gov.br/noticias/2017/regras-da-anac-para-uso-dedrones-entram-em vigor/release_drones_v2.pdf > Acesso em: 14/03/2018.

2. Amorim A, Malaman CS, Sass GG. A modernização dos processos de atualização cadastral e as análises temporais. Rev Bras Cartogr. 2013 Mar/Abr;65/2:375-382.

3. Boucher P. Domesticating the drone: The demilitarisation of unmanned aircraft for civil markets. Science and Engineering Ethics. 2015 Dez;21:1393-1412.

4. Colomina I, Molina P. Unmanned aerial systems for photogrammetry and remote sensing: A review. ISPRS Journal of Photogrammetry and Remote Sensing. 2014 Jun;92:79-97, doi:10.1016/j.isprsjprs.2014.02.013.

5. Crommelinck S, Bennett RM, Gerke M, Yang MY, Vosselman G. Contour detection for UAV-Based cadastral mapping. Journal Remote Sensing. 2017 Fev;9:1-13, doi:10.3390/rs9020171.

6. Formaggio AR, Sanches LD. Sensoriamento Remoto em Agricultura. São Paulo: Oficina de Textos; 2017. $284 \mathrm{p}$.

7. Harwin S, Lucieer A. Assessing the accuracy of georeferenced point clouds produced via multiview stereopsis from unmanned aerial vehicle (UAV) imagery. Journal Remote Sensing. 2012 Mai;4:1573-1599, doi:10.3390/rs4061573.

8. Manyoky M, Theiler P, Steudler D, Eisenbeiss H. Unmanned aerial vehicle in cadastral applications. International Archives of the Photogrammetry, Remote Sensing and Spatial Information Sciences. 2011 Set;63:1-6.

9. Maurice MJ, Koeva MN, Gerke M, Nex F, Gevaert C. A photogrammetric approach for map updating using UAV in Rwanda. In Proceedings of the GeoTech Rwanda - International Conference on Geospatial Technologies for Sustainable Urban and Rural Development. 2015 Nov;1-8.

10. Nex F, Remondino F. UAV for 3D mapping applications: a review. Applied Geomatics. 2013;6(1):115.

11. Pajares G. Overview and current status of remote sensing applications based on unmanned aerial vehicles (UAVs). Photogrammetric Engineer \& Remote Sensing. 2015 Abr;81(4):281-329, doi:10.14358/PERS.81.4.281.

12. Tahar KN; Ahmad A. An evaluation on fixed wing and multi-rotor UAV images using photogrammetric image processing. Int J Computer Inform Engineer. 2013;48-52.

13. Turner D, Lucieer A, Watson C. An automated technique for generating georectified mosaics from ultra-high resolution Unmanned Aerial Vehicle (UAV) imagery, based on Structure from Motion (SFM) point clouds. Remote Sensing. 2012 Dez;4(5):1392-1410, doi:10.3390/rs4051392. 